TRANSACTIONS OF THE

AMERICAN MATHEMATICAL SOCIETY

Volume 353, Number 9, Pages 3603-3615

S 0002-9947(01)02775-1

Article electronically published on May 4, 2001

\title{
UNIFORM PERFECTNESS OF THE LIMIT SETS OF KLEINIAN GROUPS
}

\author{
TOSHIYUKI SUGAWA
}

Dedicated to Professor Hiroki Sato on the occasion of his sixtieth birthday.

\begin{abstract}
In this note, we show, in a quantitative fashion, that the limit set of a non-elementary Kleinian group is uniformly perfect if the quotient orbifold is of Lehner type, i.e., if the space of integrable holomorphic quadratic differentials on it is continuously contained in the space of (hyperbolically) bounded ones. This result covers the known case when the group is analytically finite. As applications, we present estimates of the Hausdorff dimension of the limit set and the translation lengths in the region of discontinuity for such a Kleinian group. Several examples will also be given.
\end{abstract}

\section{INTRODUCTION}

In this note, we consider uniform perfectness of the limit sets of Kleinian groups. Once one knows that the limit set is uniformly perfect, estimation of various quantities associated with the Kleinian group becomes easier (see Section 5). Bishop and Jones effectively used this fact in their paper [4].

As soon as a proof of uniform perfectness of the limit sets of Schottky groups appeared in 2, this result was generalized to the case of finitely generated nonelementary Kleinian groups by several specialists (cf. [13] and [14]). Afterward, Canary remarked in [5] that the same result still holds for analytically finite Kleinian groups. As for Schottky groups, we should mention the pioneer work of Tsuji [19] (see the comment on Theorem $\mathrm{D}$ below).

Recently, Järvi and Vuorinen [6] proved the same result for finitely generated Kleinian groups in higher dimensional case. This is a generalization of Tukia's result 21 (geometrically finite case). It is noteworthy that their proof does not rely on Ahlfors' Finiteness Theorem.

In this note, we present a more general condition for the limit sets of Kleinian groups to be uniformly perfect (Corollary 3.3). Our method also provides a bound for uniform perfectness in terms of some geometric quantity. In practice, it is important to have such an explicit bound because the uniform perfectness connects with various quantities involving the geometry of the quotient surface (cf. [18]). Indeed, we shall give some applications in Sections 5 and 6. In Section 5, we state fundamental facts about uniform perfectness and give estimates of capacity density and Hausdorff dimension for the limit sets. Section 6 is devoted to estimation

Received by the editors June 16, 1998 and, in revised form, November 27, 2000.

2000 Mathematics Subject Classification. Primary 30F40; Secondary 30F45.

Key words and phrases. Uniformly perfect, Kleinian group, translation length, Hausdorff dimension.

(C)2001 American Mathematical Society 
of the translation length in terms of the multiplier of a loxodromic element of a Kleinian group. We shall conclude this note by giving several examples including an infinitely generated Kleinian group whose limit set is not uniformly perfect.

\section{RIEMANN ORBIFOLDS}

A 1-dimensional complex orbifold is a Hausdorff topological space locally modeled on the quotient of an open subset of the complex plane $\mathbb{C}$ under an action of a finite group whose members consist of biholomorphic maps. Note that such a finite group is necessarily a cyclic group, thus determined by its order. By a Riemann orbifold, we mean a connected 1-dimensional complex orbifold. Thus a Riemann orbifold $X$ can be regarded as a pair $(R, \nu)$ where $R$ is a Riemann surface and $\nu: R \rightarrow \mathbb{N}=\{1,2, \ldots\}$ is a multiplicity map. The value of $\nu$ corresponds to the order of the finite group, therefore, the set of singularities (branch points) $b(X)=\{x \in R ; \nu(x)>1\}$ is discrete. Set $X^{\circ}=R \backslash b(X)$. Then $X^{\circ}$ can be regarded as a subdomain of $X$ without singularities. If $\nu$ is constantly 1 , then $X$ can naturally be identified with the underlying Riemann surface $R$. For a precise definition and fundamental properties of Riemann orbifolds, we refer the reader to McMullen's book [10].

A Riemann orbifold $X=(R, \nu)$ is called hyperbolic if $R$ has a holomorphic branched Galois covering map $p: \mathbb{H} \rightarrow R$ from the upper half plane $\mathbb{H}$ onto $R$ such that $\operatorname{ord}_{\zeta} p=\nu(p(\zeta))$ for any $\zeta \in \mathbb{H}$, where $\operatorname{ord}_{\zeta} p$ denotes the local degree of $p$ at $\zeta$. The map $p$ is called a universal covering map of $X$. Hereafter, we always assume $X$ to be hyperbolic.

The covering transformation group $\Gamma=\Gamma_{X}=\{\gamma \in \operatorname{Aut}(\mathbb{H}) ; p \circ \gamma=p\}$ is called a Fuchsian model of $X$. Through the universal covering map, the Riemann orbifold $X$ inherits the hyperbolic metric $\rho_{X}$ from $\mathbb{H}$, i.e., $\rho_{\mathbb{H}}=|d z| / 2 \operatorname{Im} z=p^{*}\left(\rho_{X}\right)$. We denote by $d_{X}$ the hyperbolic distance on $X$ canonically defined by the hyperbolic metric.

Let $\mathcal{C}_{X}$ denote the set of free homotopy classes $[\alpha]=[\alpha]_{X}$ of non-trivial closed curves $\alpha$ in $X^{\circ}$. Here, a curve $\alpha$ is said to be non-trivial if it is covered by an element $\gamma \in \Gamma$ of infinite order, more precisely, if there exists a lift $\tilde{\alpha}$ via $p$ such that the initial point is translated to the terminal one by $\gamma$. A curve $\alpha$ is said to be freely homotopic to $\alpha^{\prime}$ if both curves can be covered by the same element of $\Gamma$. (The free homotopy class of a non-trivial curve precisely corresponds to the conjugacy class of an element of $\Gamma$ which covers the curve and has infinite order.) Also, let $\mathcal{C}_{X}^{*}$ be the subset of $\mathcal{C}_{X}$ consisting of free homotopy classes of curves which are covered by hyperbolic elements of $\Gamma$.

Remark 2.1. It may be more natural to consider all curves in $X$ above, however, a curve passing through a branch point of $X$ cannot be lifted via $p$ in a unique way even if the initial point is assigned. Hence being freely homotopic fails to be an equivalence relation. To avoid this difficulty, we may assign a sort of "multiplicity data" to the curve. Precisely speaking, we may consider a (regular) curve as the pair $\left(\gamma, n_{\gamma}\right)$ such that the $C^{1}$ map $\gamma: \mathrm{S}^{1} \rightarrow R$ with $\gamma^{\prime}(\zeta) \neq 0$ and $n_{\gamma}: \mathrm{S}^{1} \rightarrow \mathbb{Z}$ satisfy $0 \leq n_{\gamma}(\zeta) \leq \nu(\gamma(\zeta))-1$, where $n_{\gamma}(\zeta)$ indicates the winding number of $\gamma$ around $\gamma(\zeta)$. We, however, will not adopt this formulation here for the sake of simplicity. 
We write $\ell_{X}(\alpha)=\int_{\alpha} \rho_{X}$ and $\ell_{X}[\alpha]=\inf _{\alpha^{\prime} \in[\alpha]} \ell_{X}\left(\alpha^{\prime}\right)$. By definition, if $\gamma \in \Gamma$ covers $\alpha$, then

$$
\ell_{X}[\alpha]=l_{\gamma}=\cosh ^{-1}\left(\frac{|\operatorname{tr} \gamma|}{2}\right),
$$

where $l_{\gamma}$ denotes the translation length of $\gamma$ in $\mathbb{H}$ and $\operatorname{tr} \gamma$ is the trace of a representative of $\gamma$ in $\operatorname{SL}(2, \mathbb{R})$.

Set

$$
\begin{aligned}
L(X) & =\inf _{[\alpha] \in \mathcal{C}_{X}} \ell_{X}[\alpha]=\inf _{\gamma \in \Gamma_{X}: \text { of infinite order }} l_{\gamma}, \quad \text { and } \\
L^{*}(X) & =\inf _{[\alpha] \in \mathcal{C}_{X}^{*}} \ell_{X}[\alpha]=\inf _{\gamma \in \Gamma_{X}: \text { hyperbolic }} l_{\gamma} .
\end{aligned}
$$

We say that $X$ is of bounded geometry if $L(X)>0$. We note here that if $X$ is a Riemann surface $R$, the constant $L(R)$ is nothing but $2 I_{R}$, where $I_{R}$ is the injectivity radius of $R$. Hence, this definition agrees with the ordinary one for hyperbolic Riemann surfaces (see [18).

When the Fuchsian model $\Gamma_{X}$ of $X$ is finitely generated, the quantity $L^{*}(X)$ represents the hyperbolic length of the shortest closed geodesic in $X$. A closed geodesic which is shortest among all the curves in $X$ other than boundary curves is called a systole of $X$. Hence, $L^{*}(X)$ can be thought as the length of a systole of $X$ if $\Gamma$ is finitely generated and of the first kind. It has been recognized that the length of systole is quite an important function on the moduli space of $X$ (see [15]).

Let $A_{2}(X)$ and $B_{2}(X)$ be the complex Banach spaces consisting of holomorphic quadratic differentials $\varphi=\varphi(z) d z^{2}$ on $X$ with norms

$$
\begin{aligned}
\|\varphi\|_{1} & =\iint_{X}|\varphi|=\iint_{X}|\varphi(z)| d x d y, \\
\|\varphi\|_{\infty} & =\sup \rho_{X}^{-2}|\varphi|=\sup \rho_{X}^{-2}(z)|\varphi(z)|,
\end{aligned}
$$

respectively. The spaces $A_{2}(X)$ and $B_{2}(X)$ are canonically isomorphic to the spaces $A_{2}(\mathbb{H}, \Gamma)$ and $B_{2}(\mathbb{H}, \Gamma)$ of integrable and bounded holomorphic automorphic forms on $\mathbb{H}$ of weight -4 for $\Gamma$, respectively. We set

$$
\kappa(X)=\sup \left\{\|\varphi\|_{\infty} ; \varphi \in A_{2}(X) \text { with }\|\varphi\|_{1} \leq 1\right\} .
$$

For these spaces, the inclusion problem was first settled by Niebur and Sheingorn [12. The following strong form is due to Matsuzaki 8 .

Theorem A. The space $A_{2}(X)$ is (continuously) included in $B_{2}(X)$ if and only if $L^{*}(X)>0$. Furthermore, there exist universal constants $r_{0}$ and $r_{1}$ such that

$$
\frac{1}{2 \pi L^{*}\left(X^{\circ}\right)} \leq \kappa(X) \leq \max \left\{\frac{r_{0}}{L^{*}(X)}, r_{1}\right\}
$$

Note that the inclusion map $X^{\circ} \hookrightarrow X$ induces the restriction maps $A_{2}(X) \rightarrow$ $A_{2}\left(X^{\circ}\right)$ and $B_{2}(X) \rightarrow B_{2}\left(X^{\circ}\right)$, which are, respectively, an isometric isomorphism and a linear contraction, namely, $\|\varphi\|_{B_{2}\left(X^{\circ}\right)} \leq\|\varphi\|_{B_{2}(X)}$. The contraction follows from the monotonicity of hyperbolic metrics: $\rho_{X} \leq \rho_{X^{\circ}}$. Therefore, we have $\kappa\left(X^{\circ}\right) \leq \kappa(X)$. In fact, $\kappa\left(X^{\circ}\right) \leq \kappa(X) \leq 3 \kappa\left(X^{\circ}\right)$ also holds (see [17]).

In this article, we say that $X$ is of Lehner type if $L^{*}(X)>0$. Generally, for a (possibly disconnected) 1-dimensional complex orbifold $X$, we define $L(X)=$ $\inf L\left(X_{0}\right)$ and $L^{*}(X)=\inf L^{*}\left(X_{0}\right)$, where the infima are taken over all connected 
components $X_{0}$ of $X$, and we say that $X$ is of bounded geometry or of Lehner type if $L(X)>0$ or $L^{*}(X)>0$, respectively.

A closed set $E$ in the Riemann sphere $\widehat{\mathbb{C}}$ with $\# E \geq 3$ is called uniformly perfect if there exists a constant $c>0$ such that $E \cap\{z ; c r<|z-a|<r\} \neq \emptyset$ for all $a \in E$ and $0<r<\operatorname{diam} E$. Here $\operatorname{diam} E$ denotes the Euclidean diameter of the set $E$ with convention $\operatorname{diam} E=+\infty$ when $\infty \in E$. This is equivalent to the condition that the complement $R=\widehat{\mathbb{C}} \backslash E$ is of bounded geometry [18] (see Section 5).

\section{Kleinian GROUPS AND MAIN RESUlts}

Let $G$ be a Kleinian group, i.e., $G$ is a discrete subgroup of $\operatorname{PSL}(2, \mathbb{C})=\operatorname{Möb}(\widehat{\mathbb{C}})$ whose region of discontinuity $\Omega(G)$ in $\widehat{\mathbb{C}}$ is not empty. We denote by $\Lambda(G)$ the limit set of $G$, i.e., $\Lambda(G)=\widehat{\mathbb{C}} \backslash \Omega(G)$.

In the following, we always assume that $G$ is non-elementary, in other words, $\# \Lambda(G) \geq 3$. Then $\Lambda(G)$ is known to be a perfect set. The quotient space $X(G)=$ $\Omega(G) / G$ has a natural hyperbolic 1-dimensional complex orbifold structure with which the canonical projection $\pi: \Omega(G) \rightarrow X(G)$ is a holomorphic covering map.

Let $X$ be a connected component of $X(G)$ and $\Omega$ be a connected component of $\pi^{-1}(X)$. If $q: \mathbb{H} \rightarrow \Omega$ is a holomorphic universal covering map, then clearly $p=\pi \circ q$ is a holomorphic universal covering map of $X$. Let $H=H_{\Omega}$ be the component subgroup of $G$ corresponding to $\Omega$, i.e., $H=\operatorname{Stab}_{G}(\Omega)=\{g \in G ; g(\Omega)=\Omega\}$. We denote by $\Gamma$ and $\widetilde{\Gamma}$ the covering transformation groups of $q$ and $p$, respectively. Then, we have the natural exact sequence

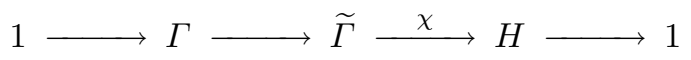

of group homomorphisms, where $\chi$ can be described as $q \circ \gamma=\chi(\gamma) \circ q$ for all $\gamma \in \widetilde{\Gamma}$.

For each $h \in H$, we denote by $l_{h, \Omega}$ the translation length of $h$ in $\Omega$ :

$$
l_{h, \Omega}:=\inf _{z \in \Omega} d_{\Omega}(z, h(z)) .
$$

Set

$$
\begin{aligned}
& H_{\mathrm{e}}=H_{\mathrm{e}, \Omega}=\{h \in H ; h \text { has a fixed point in } \Omega\}, \quad \text { and } \\
& H_{\mathrm{p}}=H_{\mathrm{p}, \Omega}=\{h \in H ; h \text { corresponds to a puncture of } X=\Omega / H\} .
\end{aligned}
$$

Precisely speaking, an element $h$ of $H$ belongs to $H_{\mathrm{p}}$ if and only if there exist an element $h_{0} \in H$ of infinite order and a subdomain $\omega$ of $\Omega$ with the following three properties:

1. $h=h_{0}^{n}$ for a non-zero integer $n$,

2. $h_{0}(\omega)=\omega$ and $g(\omega) \cap \omega=\emptyset$ for all $g \in H \backslash\left\langle h_{0}\right\rangle$, and

3. $\omega /\left\langle h_{0}\right\rangle$ is conformally equivalent to the punctured disk $\{0<|z|<1\}$.

We remark that there may exist a parabolic element $h$ of $H$ with $h \notin H_{\mathrm{p}}$ (see Example 7.2 below), while an element of $H_{\mathrm{p}} \backslash\{1\}$ is necessarily parabolic (see Proposition 6.1 below). The same thing can be said about elliptic elements. Now we define the quantities $\lambda_{H, \Omega}, \lambda_{H, \Omega}^{*}$ by

$$
\lambda_{H, \Omega}=\inf _{h \in H \backslash H_{\mathrm{e}}} l_{h, \Omega}, \quad \text { and } \quad \lambda_{H, \Omega}^{*}=\inf _{h \in H \backslash\left(H_{\mathrm{e}} \cup H_{\mathrm{p}}\right)} l_{h, \Omega}
$$


Noting that an elliptic or parabolic element in a Fuchsian group always represents a branch point or a puncture of the quotient surface, we similarly set

$$
\begin{aligned}
& \lambda_{\widetilde{\Gamma}}=\inf _{\gamma \in \widetilde{\Gamma} \backslash \widetilde{\Gamma}_{\mathrm{e}}} l_{\gamma}=\inf _{\gamma \in \widetilde{\Gamma}: \text { of infinite order }} l_{\gamma} \text { and } \\
& \lambda_{\widetilde{\Gamma}}^{*}=\inf _{\gamma \in \widetilde{\Gamma} \backslash\left(\widetilde{\Gamma}_{\mathrm{e}} \cup \widetilde{\Gamma}_{\mathrm{p}}\right)} l_{\gamma}=\inf _{\gamma \in \widetilde{\Gamma}: \text { hyperbolic }} l_{\gamma},
\end{aligned}
$$

where $l_{\gamma}$ is the translation length of $\gamma$ in $\mathbb{H}$, i.e., $l_{\gamma}=\cosh ^{-1}(|\operatorname{tr} \gamma| / 2)$. We can define $\lambda_{\Gamma}, \lambda_{\Gamma}^{*}$ in the same manner; however, $\lambda_{\Gamma}=\lambda_{\Gamma}^{*}$ holds since $\Lambda(G)$ has no isolated points. Let $N_{H, \Omega}$ be the number defined by $N_{H, \Omega}=\sup _{h \in H_{\mathrm{e}}} \operatorname{ord}(h)$, where $\operatorname{ord}(h)$ denotes the order of $h$ as an element of the group $H$. We define the number $N_{H, \Omega}^{*}$ as follows. If $H_{\mathrm{p}}$ is non-empty, then $N_{H, \Omega}^{*}=+\infty$, otherwise we set $N_{H, \Omega}^{*}=N_{H, \Omega}$.

Under these circumstances, the next result will be fundamental for our present aim.

Lemma 3.1.

$$
\begin{aligned}
& \min \left\{\frac{\lambda_{\Gamma}}{N_{H, \Omega}}, \lambda_{H, \Omega}\right\} \leq \lambda_{\widetilde{\Gamma}} \leq \min \left\{\lambda_{\Gamma}, \lambda_{H, \Omega}\right\}, \quad \text { and } \\
& \min \left\{\frac{\lambda_{\Gamma}}{N_{H, \Omega}^{*}}, \lambda_{H, \Omega}^{*}\right\} \leq \lambda_{\widetilde{\Gamma}}^{*} \leq \min \left\{\lambda_{\Gamma}, \lambda_{H, \Omega}^{*}\right\} .
\end{aligned}
$$

By the relation (2.1), we obtain $\lambda_{\Gamma}=L(\Omega), \lambda_{\widetilde{\Gamma}}=L(X)$ and the similar relations for $\lambda^{*}$ and $L^{*}$. Hence, the above statements are equivalent to the following:

$$
\begin{aligned}
& \min \left\{\frac{L(\Omega)}{N_{H, \Omega}}, \lambda_{H, \Omega}\right\} \leq L(X) \leq \min \left\{L(\Omega), \lambda_{H, \Omega}\right\}, \quad \text { and } \\
& \min \left\{\frac{L(\Omega)}{N_{H, \Omega}^{*}}, \lambda_{H, \Omega}^{*}\right\} \leq L^{*}(X) \leq \min \left\{L(\Omega), \lambda_{H, \Omega}^{*}\right\} .
\end{aligned}
$$

Now we define $\lambda(G), \lambda^{*}(G), N(G)$ and $N^{*}(G)$ by inf $\lambda_{H, \Omega}, \inf _{\Omega} \lambda_{H, \Omega}^{*}, \sup _{\Omega} N_{H, \Omega}$ and $\sup _{\Omega} N_{H, \Omega}^{*}$, respectively, where $\Omega$ runs over all components of $\Omega(G)$ and $H=$ $H_{\Omega}$. We remark here that the constants $\lambda_{H, \Omega}, \lambda_{H, \Omega}^{*}, N_{H, \Omega}$ and $N_{H, \Omega}^{*}$ depend only on the orbit of $\Omega$ under the action of $G$, i.e., on the component $X$ of $X(G)$. Then, we immediately obtain the following.

Theorem 3.2. For a non-elementary Kleinian group $G$, we have

$$
\begin{aligned}
& \min \left\{\frac{L(\Omega(G))}{N(G)}, \lambda(G)\right\} \leq L(X(G)) \leq \min \{L(\Omega(G)), \lambda(G)\}, \quad \text { and } \\
& \min \left\{\frac{L(\Omega(G))}{N^{*}(G)}, \lambda^{*}(G)\right\} \leq L^{*}(X(G)) \leq \min \left\{L(\Omega(G)), \lambda^{*}(G)\right\} .
\end{aligned}
$$

Corollary 3.3. Thus we have $L(\Omega(G)) \geq L^{*}(X(G))$. In particular, if $X(G)$ is of Lehner type, then $\Lambda(G)$ is uniformly perfect.

Corollary 3.4. For a torsion-free non-elementary Kleinian group $G$, the quotient surface $X(G)=\Omega(G) / G$ satisfies

$$
L(X(G))=\min \{L(\Omega(G)), \lambda(G)\} .
$$

We remark that the counterpart $L^{*}(X(G))=\min \left\{L(\Omega(G)), \lambda^{*}(G)\right\}$ is nothing new under the assumption that $G$ is torsion-free, parabolic-free and non-elementary.

The factors $1 / N(G)$ and $1 / N^{*}(G)$ in Theorem 3.2 seem inevitable even if $G$ is finitely generated (see Example 7.3 below). 
Remark 3.1. To guarantee that $\lambda(G)>0$ it is sufficient to assume $\sup _{z \in \Omega(G)} \iota(z)<$ $\infty$, where $\iota(z)$ denotes the injectivity radius of $\Omega(G)$ at $z$ (oral communication with Katsuhiko Matsuzaki). This condition, however, seems hard to check.

In the case that $G$ is analytically finite, i.e., $X(G)$ consists of a finite number of Riemann orbifolds of analytically finite type, the inequality $L^{*}(X(G))>0$ is easily verified. Thus we have the following corollary.

Corollary 3.5 (Canary [5]). For an analytically finite non-elementary Kleinian group $G$, the limit set $\Lambda(G)$ is uniformly perfect.

We should remark that by Ahlfors' Finiteness Theorem this result produces the finitely generated case.

\section{Proof of Lemma 3.1}

Under the same notation as in Section 3, we first prove the next elementary lemma.

Lemma 4.1. For an element $h \in H$, we have

$$
\inf _{\gamma \in \chi^{-1}(h)} l_{\gamma}=l_{h, \Omega}
$$

Proof. We recall that $\chi: \widetilde{\Gamma} \rightarrow H$ is the canonical quotient map. Let $\gamma \in \chi^{-1}(h)$, then $q \circ \gamma=h \circ q$ by definition. For a $\zeta \in \mathbb{H}$ we put $z=q(\zeta)$. Then, by definition of the hyperbolic metric and the Schwarz-Pick lemma, we have

$$
d_{\mathbb{H}}(\zeta, \gamma(\zeta)) \geq d_{\Omega}(q(\zeta), q(\gamma(\zeta)))=d_{\Omega}(z, h(z)) \geq l_{h, \Omega}
$$

Since $\zeta$ is arbitrary, we obtain $l_{\gamma} \geq l_{h, \Omega}$.

Now we prove the reverse inequality. Take a point $z$ in $\Omega$, and let $\alpha$ be a geodesic arc joining $z$ and $h(z)$ in $\Omega$ so that $d_{\Omega}(z, h(z))=\int_{\alpha} \rho_{\Omega}$. Choose a point $\zeta \in \mathbb{H}$ with $q(\zeta)=z$, and let $\beta$ be a lift of $\alpha$ via $q$ with initial point $\zeta$. Then the terminal point of $\beta$ can be written by $\gamma(\zeta)$ for some $\gamma \in \widetilde{\Gamma}$. We note here that $\chi(\gamma)=h$ by definition. Therefore, we have

$$
d_{\Omega}(z, h(z))=\int_{\alpha} \rho_{\Omega}=\int_{\beta} \rho_{\mathbb{H}} \geq d_{\mathbb{H}}(\zeta, \gamma(\zeta)),
$$

and thus $d_{\Omega}(z, h(z)) \geq l_{\gamma} \geq \inf _{\gamma \in \chi^{-1}(h)} l_{\gamma}$. Since $z$ is arbitrary, we have $l_{h, \Omega} \geq$ $\inf _{\gamma \in \chi^{-1}(h)} l_{\gamma}$. Now the proof is complete.

Now we prove Lemma 3.1 First, we note that if $\gamma \in \widetilde{\Gamma}$ is elliptic, then $h=$ $\chi(\gamma) \in H_{\mathrm{e}} \backslash\{1\}$. Note also that $\chi^{-1}(1)=\Gamma$. Therefore, we conclude that

$$
(\Gamma \backslash\{1\}) \cup \chi^{-1}\left(H \backslash H_{\mathrm{e}}\right) \subset \widetilde{\Gamma} \backslash \widetilde{\Gamma}_{\mathrm{e}},
$$

and this and the above lemma immediately yield the right-hand side inequality in (3.2).

In order to get the left-hand side inequality, we consider an element $\gamma$ of the residual part $\chi^{-1}\left(H_{\mathrm{e}} \backslash\{1\}\right) \backslash \widetilde{\Gamma}_{\mathrm{e}}$. Since $h=\chi(\gamma)$ is of finite order, say $n$, we have $\gamma^{n} \in \chi^{-1}(1)=\Gamma$, and thus $n l_{\gamma}=l_{\gamma^{n}} \geq \lambda_{\Gamma}$. Hence, $l_{\gamma} \geq \lambda_{\Gamma} / n \geq \lambda_{\Gamma} / N_{H, \Omega}$. By this observation, we are convinced of the validity of the left-hand side of (3.2). Noting that any parabolic element of $\widetilde{\Gamma}$ is mapped to $H_{\mathrm{p}}$ by the homomorphism $\chi$, we can show inequality (3.3) in the same way as above. 


\section{Some CONSEQUences}

In this section, we shall exhibit several applications of our theorems. For a closed subset $E$ of $\widehat{\mathbb{C}}$ with $\# E \geq 3$ and $\Omega=\widehat{\mathbb{C}} \backslash E$, we denote by $M_{\Omega}\left(M_{\Omega}^{\circ}\right)$ the supremum of the moduli of annuli (round annuli, respectively) separating $E$, where the modulus of an annulus is defined to be the number $m$ such that the annulus is conformally equivalent to the round annulus $\left\{z \in \mathbb{C}, 1<|z|<e^{m}\right\}$. A round annulus means a bounded annulus with boundary consisting of concentric circles. We say that an annulus $A$ separates $E$ if $A \cap E=\emptyset$ and if both components of $\widehat{\mathbb{C}} \backslash A$ intersect $E$. If $\infty \in E$, then we can define another constant $C_{\Omega}$ by $\inf _{z \in \Omega} \delta_{\Omega}(z) \rho_{\Omega}(z)$, where $\delta_{\Omega}(z)$ denotes the Euclidean distance from $z$ to $\partial \Omega$. Here it should be noted that $\delta_{\Omega}(z) \rho_{\Omega(G)}(z) \leq 1$ is always true.

With the case $E=\Lambda(G)$ and $\Omega=\Omega(G)$ in mind, we state a few results on the above constants. We can immediately deduce corresponding conclusions for Kleinian groups, however, we will not state all of those corollaries separately to avoid the repetition. The first one connects $L(\Omega)$ with $M_{\Omega}$ and $M_{\Omega}^{\circ}$.

Theorem B (cf. [18]). For an open set $\Omega$ with $\#(\widehat{\mathbb{C}} \backslash \Omega) \geq 3$, we obtain

$$
\begin{gathered}
L \leq \frac{\pi^{2}}{M_{\Omega}} \leq \min \left\{L e^{L}, \frac{1}{2} L^{2} \operatorname{coth}^{2}\left(\frac{L}{2}\right)\right\}, \quad \text { and } \\
\frac{1}{2} M_{\Omega}-K_{0} \leq M_{\Omega}^{\circ} \leq M_{\Omega},
\end{gathered}
$$

where $L=L(\Omega)$ and $K_{0}$ is an absolute constant $\leq 1.734$. Moreover, if $\infty \notin \Omega$, we also have

$$
\begin{aligned}
& M_{\Omega}-K_{1} \leq M_{\Omega}^{\circ} \leq M_{\Omega}, \quad \text { and } \\
& \frac{\tanh L / 2}{4} \leq C_{\Omega} \leq \frac{\sqrt{3} L}{\sqrt{\pi^{2}+4 L^{2}}},
\end{aligned}
$$

where $K_{1}$ is an absolute constant $\leq 2.892$.

In particular, $L(\Omega)>0$ if and only if $M_{\Omega}^{\circ}<\infty$. The first inequality in the above partly follows from the next result, which is an improvement of Maskit's theorem 7] and will be used later.

Theorem C (cf. [18]). Let $R$ be a hyperbolic Riemann surface. For the free homotopy class $[\alpha]$ of a non-trivial loop $\alpha$ in $R$, we have the estimates

$$
\ell_{R}[\alpha] \leq \frac{\pi}{2} E_{R}[\alpha] \leq \ell_{R}[\alpha] e^{\ell_{R}[\alpha]}
$$

In the above, $E_{R}[\alpha]$ denotes the extremal length of the curve family $[\alpha]$, more precisely, $E_{R}[\alpha]=\sup _{\sigma}\left(\iint_{R} \sigma(z)^{2} d x d y\right)^{-1}$, where the supremum is taken over all Borel measurable conformal metrics $\sigma$ satisfying that $\int_{\alpha^{\prime}} \sigma(z)|d z| \geq 1$ for any $\alpha^{\prime} \in$ $[\alpha]$ (such a metric $\sigma$ is called admissible for $[\alpha]$ ).

Furthermore, Pommerenke has given a remarkable characterization of uniform perfectness in terms of capacity density.

Theorem $\mathbf{D}$ (Pommerenke [13]). A closed set $E$ is uniformly perfect if and only if there exists a constant $c \in(0,1]$ such that $\operatorname{Cap}(E \cap B(a, r)) \geq$ cr for any $a \in E \backslash\{\infty\}$ and $0<r<\operatorname{diam} E$, where Cap denotes the logarithmic capacity and $B(a, r)$ denotes the closed disk centered at a with radius $r$. 
We note that $\operatorname{Cap}(B(a, r))=r$ and $2^{-7} \exp \left(-M_{\widehat{\mathbb{C}} \backslash E}^{\circ}\right)$ can be taken as the constant $c$ in the above statement (see [18]).

Here we mention the work of Tsuji. He proved in [19] that any point of the limit set of a non-elementary (finitely generated) Schottky group has a positive capacity density (with a uniform bound). In view of the above theorem, this is a substantial proof of uniform perfectness of it. Earlier than this, Myrberg [11 showed that any non-elementary Kleinian group has the limit set of positive capacity.

By virtue of Wiener's criterion, we have the following result.

Corollary 5.1. If $L(\Omega(G))>0$ the limit set $\Lambda(G)$ is regular in the sense of Dirichlet.

For general Kleinian groups, at least we can state the following.

Corollary 5.2. Let $G$ be a non-elementary Kleinian group. Any loxodromic or parabolic fixed point of $G$ is a regular point of $\Lambda(G)$ in the sense of Dirichlet.

In fact, if $z_{0}$ is a fixed point of a loxodromic or parabolic element $\gamma$ of $G$, then $\gamma$ is contained in a finitely generated non-elementary subgroup $G_{0}$ of $G$. Since $\Lambda\left(G_{0}\right)$ is uniformly perfect, by Theorem De see that $\varlimsup_{r \rightarrow 0} \operatorname{Cap}\left(\Lambda(G) \cap B\left(z_{0}, r\right)\right) / r \geq$ $\varlimsup_{r \rightarrow 0} \operatorname{Cap}\left(\Lambda\left(G_{0}\right) \cap B\left(z_{0}, r\right)\right) / r>0$, which implies the regularity of $\Lambda(G)$ at $z_{0}$ (see, for example, Tsuji 20 p. 104). Further, we should note that the set of loxodromic fixed points of a non-elementary Kleinian group is dense in the limit set.

Another application of uniform perfectness is concerned with the Hausdorff dimension. This sort of result is essentially due to Järvi-Vuorinen [6]. The following quantitative form follows from a result in [18.

Theorem E. The Hausdorff dimension $\mathrm{H}-\operatorname{dim}(E)$ of a closed set $E=\widehat{\mathbb{C}} \backslash \Omega$ can be estimated from below as follows:

$$
\mathrm{H}-\operatorname{dim}(E) \geq \frac{\log 2}{\log \left(2 e^{\left.M_{\Omega}^{\circ}+1\right)}\right.}\left(\geq \frac{\log 2}{M_{\Omega}^{\circ}+\log 3}\right) .
$$

Therefore, the information on $M_{\Omega(G)}^{\circ}$ leads to a lower estimate of the Hausdorff dimension of the limit set of $G$. As an immediate consequence of this, we can see the (known) fact that any non-elementary Kleinian group has the limit set of positive Hausdorff dimension.

\section{Estimate of TRANSLATION LENGTH}

As an application of (5.1), we present here an estimate of the translation length of a loxodromic element of a Kleinian group in the region of discontinuity in terms of the trace or the multiplier. Before stating our result, we refer to a general result on translation length, which is suggested to the author by K. Matsuzaki. The original idea is due to Bers [3]. See also the proof of Proposition 6.4 in [9].

Let $G$ be a non-elementary Kleinian group and $H$ a component subgroup of $G$ corresponding to a component $\Omega$ of the region of discontinuity $\Omega(G)$. Let $h$ be a loxodromic element of $H$, i.e., $\eta=\operatorname{tr}^{2}(h) \in \mathbb{C} \backslash[0,4]$. By the Möbius invariance of $l_{h, \Omega}$ and $\operatorname{tr}^{2}(h)$, we may assume that $h$ has the form $h(z)=\lambda z$ with $|\lambda|>1$ where $\eta=\left(\sqrt{\lambda}+\sqrt{\lambda}^{-1}\right)^{2}=\lambda+\lambda^{-1}+2$. We note that $0, \infty \in \Lambda(G)$ under this assumption. For an arbitrary $z_{0} \in \Omega$, let $\alpha$ be a geodesic arc joining $z_{0}$ and $h\left(z_{0}\right)$ in $\Omega$ such that $d_{\Omega}\left(z_{0}, h\left(z_{0}\right)\right)=\ell_{\Omega}(\alpha)$. Without loss of generality, we may further assume that $z_{0}=1$. Now we consider the quotient map $p: \mathbb{C}^{*} \rightarrow \mathbb{C}^{*} /\langle h\rangle=: T$, and 
set $R=p(\Omega)=\Omega /\langle h\rangle \subset T$. Then $\beta=p_{*} \alpha=p(\alpha)$ is a closed geodesic in $R$, where $R$ is endowed with the hyperbolic metric. We note here that $\ell_{\Omega}(\alpha)=\ell_{R}(\beta)$. In view of Theorem $\mathbb{C}$, we have $\frac{\pi}{2} E_{R}[\beta] \leq \ell_{R}[\beta] e^{\ell_{R}[\beta]}$. Since $[\beta]=[\beta]_{R} \subset[\beta]_{T}$, by the monotonicity of extremal length (cf. [1]), it turns out that $E_{R}[\beta] \geq E_{T}[\beta]_{T}$. Thus it is sufficient to compute $E_{T}[\beta]_{T}$.

The function $q(z)=\exp (2 \pi i z)$ is a universal covering map of $\mathbb{C}^{*}$ from the complex plane $\mathbb{C}$. Let $\tilde{\alpha}$ be a lift of $\alpha$ via $q$ with initial point 0 and let $\tau$ denote the terminal point of $\tilde{\alpha}$. In particular, $\exp (2 \pi i \tau)=\lambda$, so we may write $\tau=\frac{1}{2 \pi i} \log \lambda$. Further, note that $p \circ q: \mathbb{C} \rightarrow T$ is a quotient map of $\mathbb{C}$ by the lattice generated by 1 and $\tau$ over $\mathbb{Z}$. Then, each curve in $[\beta]_{T}$ is lifted to an arc with initial point, say $a$, in $[0,1]$ and with terminal point $a+\tau$, and vice versa. Thus, by the standard length-area method (see [1]), one can easily see that the extremal admissible metric for $[\beta]_{T}$ is given by the projection $\sigma$ of $|\tau|^{-1}|d z|$ under $p \circ q$, and hence $E_{T}[\beta]_{T}=\left(\iint_{T} \sigma^{2}\right)^{-1}=\left(|\tau|^{-2} \cdot|\operatorname{Im} \tau|\right)^{-1}=|\tau|^{2} /|\operatorname{Im} \tau|=|\operatorname{Im}(1 / \tau)|^{-1}$. Thus we have

$$
\ell_{R}[\beta] e^{\ell_{R}[\beta]} \geq \frac{\pi|\tau|^{2}}{2|\operatorname{Im} \tau|}=\frac{|\log \lambda|^{2}}{4 \log |\lambda|} .
$$

The quantity $\log \lambda$ is sometimes called the complex length of $h$ (cf. 9]). Here, we denote by $\log z$ the principal branch of $\log z$, i.e., $\log z$ is the branch of the $\operatorname{logarithm}$ determined by $-\pi<\operatorname{Im} \log z \leq \pi$. Since $|\log \lambda| \geq|\log \lambda|$ and $z_{0}$ is arbitrary, we have the following.

Proposition 6.1. Let $G$ be a non-elementary Kleinian group and $H$ be its component subgroup corresponding to a component $\Omega$ of $\Omega(G)$. For any loxodromic element $h$ of $H$ with multiplier $\lambda(|\lambda|>1)$ we have the estimate of the translation length of $h$ in $\Omega$ :

$$
l_{h, \Omega} \exp \left(l_{h, \Omega}\right) \geq \frac{|\log \lambda|^{2}}{4 \log |\lambda|}\left(\geq \frac{1}{4} \log |\lambda|\right) .
$$

The left-hand side of this inequality is of exponential order, and it seems difficult to improve this order in general. However, if the limit set $\Lambda(G)$ is uniformly perfect, we could have an estimate of linear order.

Theorem 6.2. Let $H$ be a component subgroup of a non-elementary Kleinian group $G$ corresponding to a component $\Omega$ of $\Omega(G)$, and suppose that $C=\frac{1}{4} \tanh (L(\Omega) / 2)$ $>0$. Then, for a loxodromic element $h \in H$, the translation length $l_{h, \Omega}$ can be estimated by

$$
l_{h, \Omega} \geq C|\log \lambda|
$$

where $\lambda$ is the multiplier of $h$, i.e., $\operatorname{tr}^{2}(h)=\lambda+\lambda^{-1}+2$.

Remark 6.1. Noting the inequality $C \geq \frac{1}{4} \tanh (L(\Omega(G)) / 2)$, we can see that $C>0$ if $\Lambda(G)$ is uniformly perfect. When $\Omega$ is simply connected, $L(\Omega)=\infty$ by definition. Hence, we have $l_{h, \Omega} \geq|\log \lambda| / 4$.

We further remark that, in general, we cannot estimate the translation length from above by the trace or multiplier. This can be understood by the existence of accidental parabolic transformations.

Proof of Theorem 6.2. We shall prove the theorem under exactly the same normalizations and notation in the previous sentences. If we denote by $\delta(z)$ the distance 
from $z$ to $\Lambda(G)$, by (5.1), we have $\delta(z) \rho_{\Omega}(z) \geq C$ for $z \in \Omega$ and $\delta(z) \leq|z|$ because $0 \in \Lambda(G)$. Then, we compute

$$
\begin{aligned}
d_{\Omega}\left(z_{0}, h\left(z_{0}\right)\right) & =\int_{\alpha} \rho_{\Omega}(z)|d z| \geq C \int_{\alpha} \frac{|d z|}{\delta(z)} \geq C \int_{\alpha} \frac{|d z|}{|z|} \\
& =C \int_{\tilde{\alpha}}|d \zeta| \geq C\left|\int_{\tilde{\alpha}} d \zeta\right|=C|\log \lambda| \geq C|\log \lambda| .
\end{aligned}
$$

Now the theorem has been proved.

\section{EXAMPLES}

In this section, we present simple examples of Kleinian groups of Schottky type. The first construction provides an example of an infinitely generated Kleinian group whose limit set is not uniformly perfect. Looking at Theorem 3.2, one may guess that it is sufficient to construct a Kleinian group whose quotient orbifold has arbitrarily short geodesics which are lifted to closed curves in the region of discontinuity. In fact, such an example can be given by infinitely generated Schottky groups as Pommerenke indicated in [13].

Example 7.1. Let $a_{j}, b_{j} \in \mathbb{C}$ be sequences tending to $\infty$, and $r_{j}>0$ and $\alpha_{j} \in \mathbb{C}$ with $\left|\alpha_{j}\right|=1$ be given so that all closed disks $A_{j}=B\left(a_{j}, r_{j}\right), B_{j}=B\left(b_{j}, r_{j}\right)$ are disjoint $(j=1,2, \ldots)$. Then $A_{j}$ and $B_{j}$ are the isometric circles of the Möbius transformations $g_{j}$ and $g_{j}^{-1}$, respectively, where $g_{j}(z)=b_{j}-\frac{\alpha_{j} r_{j}^{2}}{z-a_{j}}$. Thus $G=$ $\left\langle g_{1}, g_{2}, \ldots\right\rangle$ is an infinitely generated Schottky group with fundamental domain $\mathbb{C} \backslash \bigcup_{j}\left(A_{j} \cup B_{j}\right) \subset \Omega(G)$.

Set $\tilde{r}_{j}=\operatorname{dist}\left(a_{j},\left(\bigcup_{k \neq j} A_{k}\right) \cup\left(\bigcup_{k} B_{k}\right)\right)>r_{j}$. Then, we can directly see that $M_{\Omega(G)}^{\circ} \geq \sup _{j} \tilde{r}_{j} / r_{j}$, and hence $\Lambda(G)$ is not uniformly perfect if $\sup _{j} \tilde{r}_{j} / r_{j}=\infty$.

The second construction serves an infinitely generated Kleinian group $G$ which contains a parabolic element $h$ which does not represent any puncture of $\Omega(G) / G$.

Example 7.2. Let $h$ be the transformation $z \mapsto z+2 i$. For $j \in \mathbb{Z}$, we take $a_{j}, b_{j} \in \mathbb{R}$ and $0<r_{j}<1$ in such a way that $b_{j}-a_{j}>2 r_{j}$ and $a_{j+1}-b_{j}>r_{j}+r_{j+1}$ and $\lim _{j \rightarrow \pm \infty} a_{j}= \pm \infty$. Taking a sequence $\alpha_{j}$ in the unit circle, we set $g_{j}(z)=b_{j}-\frac{\alpha_{j} r_{j}^{2}}{z-a_{j}}$. Then the domain $\omega=\{z \in \mathbb{C} ;|\operatorname{Im} z|<1\} \backslash \bigcup_{j}\left(A_{j} \cup B_{j}\right)$ is a fundamental domain of the Kleinian group $G$ with free generators $h, g_{j}(j \in \mathbb{Z})$, where $A_{j}=B\left(a_{j}, r_{j}\right)$ and $B_{j}=B\left(b_{j}, r_{j}\right)$. In particular, one can observe that the Riemann surface $\Omega(G) / G$ has no punctures. Note that $\Lambda(G)$ is a Cantor set and that $\Omega(G)$ is connected.

Let $\beta_{j}:[-1,1] \rightarrow \Omega(G)$ be the curve given by $\beta_{j}(t)=t i+\left(a_{j}+b_{j}\right) / 2$. Since $\delta\left(\beta_{j}(t)\right) \geq\left(b_{j}-a_{j}\right) / 2-r_{j}$, we have

$$
\ell_{\Omega(G)}\left(\beta_{j}\right) \leq \int_{\beta_{j}} \frac{|d z|}{\delta(z)} \leq \frac{4}{b_{j}-a_{j}-2 r_{j}},
$$

where $\delta(z)$ denotes the Euclidean distance from $z$ to $\Lambda(G)$.

Since $h\left(\beta_{j}(-1)\right)=\beta_{j}(1)$, we see that $l_{h, \Omega(G)} \leq \inf _{j \in \mathbb{Z}} \ell_{\Omega(G)}\left(\beta_{j}\right)$. Therefore, if $\sup _{j}\left(b_{j}-a_{j}\right)=\infty$, then we obtain $l_{h, \Omega(G)}=0$. On the other hand, if $r_{j}=$ $r_{0}, \alpha_{j}=\alpha_{0}, a_{j}=a_{0}+2 j\left(b_{0}-a_{0}\right)$ and $b_{j}=b_{0}+2 j\left(b_{0}-a_{0}\right)$ for all $j \in \mathbb{Z}$, then $G$ is a normal subgroup of the Kleinian group $\widetilde{G}=\left\langle h, h^{*}, g_{0}\right\rangle$, where $h^{*}(z)=$ $z+2\left(b_{0}-a_{0}\right)$. The property $G \triangleleft \widetilde{G}$ implies $\Omega(\widetilde{G})=\Omega(G)$. Since $X(\widetilde{G})=\Omega(G) / \widetilde{G}$ 
is a compact Riemann surface of genus 2 , we obtain $L(X(\widetilde{G}))>0$. Thus we have $\lambda(G) \geq L(X(G)) \geq L(X(\widetilde{G}))>0$. In particular, $l_{h, \Omega(G)}>0$ in this case.

Finally, we give a family of finitely generated, torsion-free (actually, free) Kleinian groups which shows that the factor $1 / N^{*}(G)$ is likely to be necessary in (3.5).

Example 7.3. Fix $0<r<1$ and $\alpha \in \mathbb{C}$ with $|\alpha|=1$. Set $h(z)=z+2 i$ and $g_{t}(z)=2 t-\alpha r^{2} / z$, where $t>1$ is a real parameter. Then $G_{t}=\left\langle h, g_{t}\right\rangle$ is a free Kleinian group with fundamental domain $\omega=\hat{\omega} \backslash\left(B \cup B^{\prime}\right)$, where $\hat{\omega}=\{z \in$ $\mathbb{C} ;|\operatorname{Im} z|<1\}, B=\{|z| \leq r\}$ and $B^{\prime}=\{|z-2 t| \leq r\}$. Since $h$ represents a pair of punctures of the quotient surface, the element $h$ is contained in $G_{\mathrm{p}}$. Note also that $N^{*}\left(G_{t}\right)=+\infty$ in this case. We now prove the following claim: There exist constants $K<+\infty$ and $c>0$ depending only on $r$ such that

$$
\begin{gathered}
c \leq \min \left\{L\left(\Omega\left(G_{t}\right)\right), \lambda^{*}\left(G_{t}\right)\right\} \quad \text { and } \\
L^{*}\left(X\left(G_{t}\right)\right) \leq \frac{K}{t} .
\end{gathered}
$$

Therefore, we would never have a general lower estimate of the form $L^{*}(X(G)) \geq$ $c_{0} \min \left\{L(\Omega(G)), \lambda^{*}(G)\right\}$ for some positive absolute constant $c_{0}$.

Let us write $g=g_{t}, G=G_{t}$ and $\Omega=\Omega\left(G_{t}\right)$. We begin with a few preliminary observations. We set $B_{n}=h^{n}(B), B_{n}^{\prime}=h^{n}\left(B^{\prime}\right)$ and $\Lambda_{n}=B_{n} \cap \Lambda(G), \Lambda_{n}^{\prime}=$ $B_{n}^{\prime} \cap \Lambda(G)$ for $n \in \mathbb{Z}$. Then we know that $\Lambda(G) \backslash\{\infty\}=\bigcup_{n \in \mathbb{Z}}\left(\Lambda_{n} \cup \Lambda_{n}^{\prime}\right)$. Since $\infty \in \Lambda(G), 0=g^{-1}(\infty)$ and $2 t=g(\infty)$ belong to $\Lambda(G)$. For later use, we estimate the quantity $d=\sup \left\{|z| ; z \in \Lambda_{0}\right\}$. First we note

$$
d=\sup _{w \in \Lambda(G) \backslash B^{\prime}}\left|g^{-1}(w)\right|=\sup _{w \in \Lambda(G) \backslash B^{\prime}} \frac{r^{2}}{|w-2 t|}=\frac{r^{2}}{\operatorname{dist}\left(2 t, \Lambda(G) \backslash B^{\prime}\right)} .
$$

Since $2-r \leq \operatorname{dist}\left(2 t, \Lambda(G) \backslash B^{\prime}\right) \leq 2$, we obtain $r^{2} / 2 \leq d \leq r^{2} /(2-r)<r^{2}$. In particular, we see

$$
\operatorname{dist}\left(\partial B, \Lambda_{0}\right) \geq 1-r^{2} .
$$

Noting $g^{-1}(2 t \pm 2 i)= \pm \alpha r^{2} i / 2 \in \Lambda(G)$, we also have $\operatorname{diam} \Lambda_{0} \geq r^{2}$.

For simplicity, we further assume that $0<r \leq 1 / 2$ in the sequel. First we prove $L(\Omega) \geq c_{1}>0$ for a constant $c_{1}$ depending only on $r$. To see this, it suffices to show that $M_{\Omega}^{\circ}$ is uniformly bounded by Theorem $\mathrm{B}$. Let $A$ be an arbitrary round annulus in $\Omega$ which separates $\Lambda(G)$. Set $\rho=\exp (-m(A))$, where $m(A)$ denotes the modulus of $A$. We note here the following elementary lemma.

Lemma 7.1. For the round annulus $A=\{\rho<|z|<1\}$, we set $A_{0}=\left\{\rho \frac{3+\rho}{1+3 \rho}<\right.$ $\left.|z|<\frac{1+3 \rho}{3+\rho}\right\}$. Then, if a Möbius transformation $T$ maps $A$ into $\mathbb{C}$, we can take a round annulus $A^{\prime}$ in $T(A)$ in such a way that $T\left(A_{0}\right) \subset A^{\prime}$. In particular, $m\left(A^{\prime}\right) \geq$ $\log 1 / \rho+2 \log \frac{1+3 \rho}{3+\rho} \geq m(A)-2 \log 3$.

Now we take the annulus $A_{0}$ in $A$ with the property similar to the above, and choose an element $f \in G$ such that $f\left(A_{0}\right) \cap \omega \neq \emptyset$. Then $f(A)$ contains a round annulus $A^{\prime}=\left\{z ; r_{0}<|z-a|<r_{1}\right\}$ with $f\left(A_{0}\right) \subset A^{\prime}$. We note here that $\log r_{1} / r_{0}=$ $m\left(A^{\prime}\right) \geq m\left(A_{0}\right) \geq m(A)-2 \log 3$. Now we estimate $r_{1} / r_{0}$ from above. Since $A^{\prime}$ separates $\Lambda(G)$, we have $r_{1}-r_{0} \leq 2$, and hence $r_{1} / r_{0} \leq 1+2 / r_{0}$. By construction, the closed disk $E=\left\{z ;|z-a| \leq r_{0}\right\}$ intersects $\Lambda_{n}=B_{n} \cap \Lambda(G)$ or $\Lambda_{n}^{\prime}=B_{n}^{\prime} \cap \Lambda(G)$ for some $n \in \mathbb{Z}$. Conjugating by $h^{n}$, we may assume that $E$ intersects $\Lambda_{0}$ or $\Lambda_{0}^{\prime}$. 
Assume that $E \cap \Lambda_{0} \neq \emptyset$. (The other case can be treated similarly, so we omit it.) We further divide the case into three parts.

1. In the case $E \cap \Lambda(G) \backslash \Lambda_{0} \neq \emptyset$, we have $2 r_{0} \geq 2-2 r^{2}$, and thus $r_{1} / r_{0} \leq$ $1+2 /\left(1-r^{2}\right)<5$.

2. In the case $E \cap \Lambda(G)=\Lambda_{0}$, we have $2 r_{0} \geq \operatorname{diam} \Lambda_{0} \geq r^{2}$, and thus $r_{1} / r_{0} \leq$ $1+4 / r^{2}$.

3. In the case $E \cap \Lambda(G) \subsetneq \Lambda_{0}$, the annulus $A^{\prime}$ separates $\Lambda_{0}$, and thus $r_{1}-r_{0} \leq$ $\operatorname{diam} \Lambda_{0} \leq 2 r^{2}$. On the other hand, by assumption, we can take points $z \in$ $\omega \cap A^{\prime}$ and $w \in E \cap \Lambda(G)$. Then $r_{1}+r_{0} \geq|z-w|>\operatorname{dist}(\partial B, B \cap \Lambda(G)) \geq 1-r^{2}$. By these inequalities, we see $r_{1} / r_{0} \leq\left(1+r^{2}\right) /\left(1-3 r^{2}\right) \leq 5$.

In any case, $\exp (m(A)) / 9 \leq r_{1} / r_{0} \leq 1+4 / r^{2}$ holds provided that $r \leq 1 / 2$. Hence we have $M_{\Omega}^{\circ} \leq \log \left(1+4 / r^{2}\right)+2 \log 3$, which implies $L(\Omega) \geq c_{1}=c_{1}(r)>0$.

In particular, by Theorem $\mathbb{B}, \delta(z) \rho_{\Omega}(z) \geq C_{1}$ holds for $z \in \Omega$, where $\delta(z)=$ $\operatorname{dist}(z, \partial \Omega)$ and $C_{1}$ is a positive constant depending only on $r$. Using this fact, we next show $\lambda^{*}(G) \geq c_{2}>0$. We first prepare the following lemma.

Lemma 7.2. The hyperbolic distance between $\partial B$ and $\partial \omega \backslash \partial B$ in $\Omega$ is at least $C_{1} \log 1 / r$.

Proof. Let $z_{1} \in \partial B$ and $z_{2} \in \partial \omega \backslash \partial B$. Note $\left|z_{1}\right|=r$ and $\left|z_{2}\right| \geq 1$. Taking a geodesic arc $\alpha$ joining $z_{1}$ and $z_{2}$, we have $d_{\Omega}\left(z_{1}, z_{2}\right)=\int_{\alpha} \rho_{\Omega} \geq C_{1} \int_{\alpha}|d z| / \delta(z) \geq$ $C_{1} \int_{\alpha}|d z| /|z| \geq C_{1} \int_{\left|z_{1}\right|}^{\left|z_{2}\right|} d s / s \geq C_{1} \log 1 / r$, where we used the fact $\delta(z) \leq|z|$. Since $z_{1}$ and $z_{2}$ are arbitrary, we have the conclusion.

Now let $z_{0}$ be an arbitrary point in $\Omega$ and let $f$ be an arbitrary element of $G \backslash\left(G_{\mathrm{p}} \cup G_{\mathrm{e}}\right)$. In this case, any parabolic element in $G$ is conjugate to a power of $h$. We see therefore that $f$ is loxodromic and the two fixed points, say $a, b$, of $f$ are both finite. Now take a geodesic arc $\alpha$ connecting $z_{0}$ and $f\left(z_{0}\right)$ in $\Omega$ so that $d_{\Omega}\left(z_{0}, f\left(z_{0}\right)\right)=\ell_{\Omega}(\alpha)$. Then $\hat{\alpha}=\bigcup_{n \in \mathbb{Z}} f^{n}(\alpha)$ is an open arc in $\Omega$ with endpoints $a$ and $b$. Since $f$ is not conjugate to $h$, we can choose an element $k \in G$ such that a component, say $\beta$, of $k(\hat{\alpha}) \cap \omega$ connects $\partial B$ or $\partial B^{\prime}$ with another boundary of $\omega$. We may assume that $\beta$ connects $\partial B$ with another boundary of $\omega$. Noting the invariance of $\rho_{\Omega}$ under $G$, by the above lemma, we obtain

$$
d_{\Omega}\left(z_{0}, f\left(z_{0}\right)\right)=\int_{\alpha} \rho_{\Omega} \geq \int_{\beta} \rho_{\Omega} \geq C_{1} \log 1 / r .
$$

Thus, we obtain $\lambda^{*}(G) \geq C_{1} \log 1 / r=: c_{2}$. Inequality (7.1) is now obtained by taking $c=\min \left\{c_{1}, c_{2}\right\}$.

Finally, we show inequality (7.2). Let $\beta:[-1,1] \rightarrow \Omega$ be the curve given by $\beta(s)=t+s i$. Then the image of $\beta$ in $X(G)$ is a non-trivial closed curve which is not homotopic to any puncture. Therefore, by the same method as in Example 7.2 we have

$$
L^{*}(X(G)) \leq \ell_{\Omega}(\beta) \leq \frac{2}{t-r} \leq \frac{K}{t}
$$

where $K=2 /(1-r)$.

Remark 7.1. By approximating the above $h$ by suitable elliptic transformations, we could have similar examples which show that the factor $1 / N(G)$ also seems necessary in (3.4). 


\section{ACKNOWLEDGEMENT}

The author wishes to express his gratitude to Katsuhiko Matsuzaki for telling him the main idea of Proposition 6.1.

After submission, the author learned of the interesting paper [16], in which a simple proof is given for the uniform perfectness of the limit set of a Kleinian group in a different context. However, as is mentioned in that paper, his result can actually be applicable only for finitely generated Kleinian groups. The author is grateful to Rich Stankewitz for drawing the author's attention to this paper and for helpful comments.

\section{REFERENCES}

1. L. V. Ahlfors, Lectures on Quasiconformal Mappings, van Nostrand, 1966. MR 34:336

2. A. F. Beardon and Ch. Pommerenke, The Poincaré metric of plane domains, J. London Math. Soc. (2) 18 (1978), 475-483. MR 80a:30020

3. L. Bers, On boundaries of Teichmüller spaces and on Kleinian groups: I, Ann. of Math. (2) 91 (1970), 570-600. MR 45:7044

4. C. J. Bishop and P. W. Jones, Hausdorff dimension and Kleinian groups, Acta Math. 179 (1997), 1-39. MR 98k:22043

5. R. D. Canary, The Poincaré metric and a conformal version of a theorem of Thurston, Duke Math. J. 64 (1991), 349-359. MR 92k:57020

6. P. Järvi and M. Vuorinen, Uniformly perfect sets and quasiregular mappings, J. London Math. Soc. 54 (1996), 515-529. MR 98d:30031

7. B. Maskit, Comparison of hyperbolic and extremal lengths, Ann. Acad. Sci. Fenn. Ser. A I Math. 10 (1985), 381-386. MR 87c:30062

8. K. Matsuzaki, Bounded and integrable quadratic differentials: hyperbolic and extremal lengths on Riemann surfaces, Geometric Complex Analysis (J. Noguchi et al., ed.), World Scientific, Singapore, 1996, pp. 443-450. MR 98h:30060

9. C. McMullen, Iteration on Teichmüller space, Invent. Math. 99 (1990), 425-454. MR 91a:57008

10. Complex Dynamics and Renormalization, Ann. of Math. Studies, Princeton, 1994. MR 96b:58097

11. P. J. Myrberg, Die Kapazität der singulären Menge der linearen Gruppen, Ann. Acad. Sci. Fenn. A I Math.-Phys. 10 (1941), 1-19. MR 7:516c

12. D. Niebur and M. Sheingorn, Characterization of Fuchsian groups whose integrable forms are bounded, Ann. of Math. (2) 106 (1977), 239-258. MR 57:6411

13. Ch. Pommerenke, Uniformly perfect sets and the Poincaré metric, Arch. Math. 32 (1979), 192-199. MR 80j:30073

14. __ On uniformly perfect sets and Fuchsian groups, Analysis 4 (1984), 299-321. MR 86e: 30044

15. P. Schmutz Schaller, Systoles and topological Morse functions for Riemann surfaces, J. Differential Geom. 52 (1999), 407-452. CMP 2000:13

16. R. Stankewitz, Uniformly perfect sets, rational semigroups, Kleinian groups and IFS's, Proc. Amer. Math. Soc. 128 (2000), 2569-2575. CMP 2000:44

17. T. Sugawa, On the geometry of hyperbolic 2-orbifolds, preprint

18. Warious domain constants related to uniform perfectness, Complex Variables Theory Appl. 36 (1998), 311-345. MR 99h:30043

19. M. Tsuji, On the capacity of general Cantor sets, J. Math. Soc. Japan 5 (1953), 235-252. MR 15:309h

20. - Potential Theory in Modern Function Theory, Maruzen, Tokyo, 1959. MR 22:5712

21. P. Tukia, On limit sets of geometrically finite groups, Math. Scand. 57 (1985), 29-43. MR 87d:30045

Department of Mathematics, Kyoto University, 606-8502 Kyoto, Japan

Current address: Department of Mathematics, University of Helsinki, P. O. Box 4 (Yliopistonkatu 5), FIN-00014, Helsinki, Finland

E-mail address: sugawa@kusm.kyoto-u.ac.jp 